\title{
CALR NP_004334.1:p.L367fs*46
}

National Cancer Institute

\section{Source}

National Cancer Institute. CALR NP 004334.1:p.L367fs*46. NCI Thesaurus, Code C129837.

A change in the amino acid composition of the calreticulin protein where a frameshift mutation results in the insertion of 46 non-canonic amino acids followed by a stop codon immediately after the leucine residue at position 367. 